\title{
Functional genetic variants in complement component 7 confer susceptibility to gastric cancer
}

\author{
Siyue Wang ${ }^{1,2}$, Wenqian Hu ${ }^{1}$, Yuning Xie ${ }^{1}$, Hongjiao Wu ${ }^{1}$, Zhenxian Jia ${ }^{1}$, Zhi Zhang ${ }^{3}$, Xuemei Zhang ${ }^{\text {Corresp. } 1,2}$ \\ ${ }^{1}$ School of Public Health, North China University of Science and Technology, Tangshan, China \\ 2 College of Life Science, North China University of Science and Technology, Tangshan, China \\ 3 Affiliated Tangshan Gongren Hospital, North China University of Science and Technology, Tangshan, China \\ Corresponding Author: Xuemei Zhang \\ Email address: jyxuemei@gmail.com
}

Background Complement system plays an important role in innate immunity which involved in the changes tumor immune microenvironment by mediating the inflammatory response. This study aims to explore the relationship between complement component 7 (C7) polymorphisms and the risk of gastric cancer (GC). Materials and methods All selected SNPs of $C 7$ were genotyped in 471 patients and 471 controls using the polymerase chain reaction-restriction fragment length polymorphism (PCR-RFLP). Odds ratios (ORs) and 95\% confidence intervals (C/s) were calculated by unconditional Logistic regression to analyze the relationship between each genotype and the genetic susceptibility to gastric cancer. The level of $C 7$ expression in GC was analyzed by Gene Expression Profiling Interactive Analysis (GEPIA) and detected by Enzyme Linked Immunosorbent Assay. Kaplan-Meier plotter were used to reveal $C 7$ of prognostic value in GC. We examined SNPs association with the expression of $C 7$ using the GTEx database. The effect of $C 7$ polymorphisms on the regulatory activity of $C 7$ was detected by luciferase reporter assay. Results Unconditional logistic regression showed that individuals with $C 7$ rs1376178 AA or CA genotype had a higher risk of GC with OR $(95 \% \mathrm{Cl})$ of 2.09 (1.43-3.03) and 1.88(1.35-2.63), respectively. For C7 rs1061429 C>A polymorphism, AA genotype was associated with the elevated risk for developing gastric cancer $(\mathrm{OR}=2.16,95 \% \mathrm{Cl}=$ 1.37-3.38). In stratified analysis, C7 rs1376178 AA genotype increased the risk of GC among males $(\mathrm{OR}=2.88,95 \% \mathrm{Cl}=1.81-4.58)$, but not among females $(\mathrm{OR}=1.06,95 \% \mathrm{Cl}$ $=0.55-2.06)$. Individuals carrying rs 1061429 AA significantly increased the risk of gastric cancer among youngers $(\mathrm{OR}=2.84,95 \% \mathrm{Cl}=1.39-5.80)$ and non-smokers $(\mathrm{OR}=2.79$, $95 \% \mathrm{Cl}=1.63-4.77) . C 7$ was overexpressed in gastric cancer tissues and serum of cancer patients and was significantly associated with the prognosis. C7 rs1061429 C>A variant contributed to reduced protein level of $C 7(P=0.029)$, but rs1376178 didn't. Luciferase reporter assay showed that rs1376178C-containing plasmid exhibited 2.86-fold higher 
luciferase activity than rs1376178 A-containing plasmid $(P<0.001)$. We also found that rs1061429A allele contributed 1.34-fold increased luciferase activity than rs1061429C allele when co-transfected with miR-591 $(P=0.0012)$. Conclusions These findings highlight the role of $C 7$ in the development of gastric cancer. 
1 Functional genetic variants in complement component 7 confer susceptibility to gastric

2 cancer

3 Siyue Wang ${ }^{1,2}$, Wenqian $\mathrm{Hu}^{1}$, Yuning Xie ${ }^{1}$, Hongjiao $\mathrm{Wu}^{1}$, Zhenxian $\mathrm{Jia}^{1}$, Zhi Zhang ${ }^{3}$, Xuemei

4 Zhang $1,2 *$

5

$6{ }^{1}$ School of Public Health, North China University of Science and Technology, Tangshan, China

$7 \quad{ }^{2}$ College of Life Science, North China University of Science and Technology, Tangshan, China

$8{ }^{3}$ Affiliated Tangshan Gongren Hospital, North China University of Science and Technology,

9 Tangshan, China

10

11 * Corresponding author: Xuemei Zhang. North China University of Science and Technology,

12 Tangshan, China

13 E-mail: jyxuemei@gmail.com 
ABSTRACT

Background Complement system plays an important role in innate immunity which involved in the changes tumor immune microenvironment by mediating the inflammatory response. This study aims to explore the relationship between complement component $7(C 7)$ polymorphisms and the risk of gastric cancer (GC). Materials and methods All selected SNPs of $C 7$ were genotyped in 471 patients and 471 controls using the polymerase chain reaction-restriction fragment length polymorphism (PCR-RFLP). Odds ratios (ORs) and 95\% confidence intervals (CIs) were calculated by unconditional Logistic regression to analyze the relationship between each genotype and the genetic susceptibility to gastric cancer. The level of $C 7$ expression in GC was analyzed by Gene Expression Profiling Interactive Analysis (GEPIA) and detected by Enzyme Linked Immunosorbent Assay. Kaplan-Meier plotter were used to reveal $C 7$ of prognostic value in GC. We examined SNPs association with the expression of $C 7$ using the GTEx database. The effect of $C 7$ polymorphisms on the regulatory activity of $C 7$ was detected by luciferase reporter assay. Results Unconditional logistic regression showed that individuals with $C 7$ rs1376178 AA or CA genotype had a higher risk of GC with OR (95\%CI) of 2.09 (1.433.03) and 1.88(1.35-2.63), respectively. For C7 rs1061429 C>A polymorphism, AA genotype was associated with the elevated risk for developing gastric cancer $(\mathrm{OR}=2.16,95 \% C I=1.37$ 3.38). In stratified analysis, $C 7 \mathrm{rs} 1376178$ AA genotype increased the risk of GC among males $(\mathrm{OR}=2.88,95 \% C I=1.81-4.58)$, but not among females $(\mathrm{OR}=1.06,95 \% C I=0.55-2.06)$ Individuals carrying rs1061429 AA significantly increased the risk of gastric cancer among youngers $(\mathrm{OR}=2.84,95 \% \mathrm{CI}=1.39-5.80)$ and non-smokers $(\mathrm{OR}=2.79,95 \% \mathrm{CI}=1.63-4.77) . C 7$ was overexpressed in gastric cancer tissues and serum of cancer patients and was significantly associated with the prognosis. $C 7 \mathrm{rs} 1061429 \mathrm{C}>\mathrm{A}$ variant contributed to reduced protein level of 
$38 C 7(P=0.029)$, but rs1376178 didn't. Luciferase reporter assay showed that rs $1376178 \mathrm{C}-$

39 containing plasmid exhibited 2.86-fold higher luciferase activity than rs1376178 A-containing

40 plasmid $(P<0.001)$. We also found that rs1061429A allele contributed 1.34-fold increased

41 luciferase activity than rs 1061429C allele when co-transfected with miR-591 $(P=0.0012)$.

42 Conclusions These findings highlight the role of $C 7$ in the development of gastric cancer.

44 Key words $C 7$, Gastric cancer, Genetic variant, Single nucleotide polymorphism 


\section{INTRODUCTION}

46 Gastric cancer is one of the common malignant cancer worldwide, especially in East Asia(Siegel

47 et al. 2021). The incidence of gastric cancer ranks the third and fourth among men and women in

48 China, respectively (Chen et al. 2016). For most gastric cancer patients, more symptoms are

49 usually considered to be related to an advanced stage and the surgical resection is still the main

50 therapeutic choice (Digklia \& Wagner 2016; Song et al. 2017). Several Genome-wide association studies (GWAS) datasets showed that genetic variants were significantly associated

52 with gastric cancer risk (Jin et al. 2020; Saeki et al. 2013).

53 The human immune system is made up of two distinct parts, innate immune system and adaptive immune system. As the body's first line of defense against germs and foreign substances, innate immune system provides immediate and non-specific immune responses, which is different from the way in which adaptive immune system specifically recognizes and eliminates pathogens

57 through specialized T and B lymphocytes (Berraondo et al. 2016; Saeki et al. 2013).

Complement system is a critical component of innate immunity, which can be activated by three major pathways: the classical pathway, the alternative pathway, and the Mannose-binding Lectin (MBL) pathway. Many studies have demonstrated that complement activation enhances innate immunity against cancer through immune infiltrating or complement-dependent cytotoxicity (Bao et al. 2020; Park et al. 2012). In addition, complement components $C 5 a$ and $C 3 a$ generated by complement cascades facilitate cancer cell proliferation and regeneration (Markiewski et al. 2008; Ostrand-Rosenberg 2008). 
66 the final product of the complement cascade (Würzner 2000), which acts as one of major rate-

67 limiting factors for the formation of membrane attack complex (MAC) (Walport 2001; Ying et

68

69 al. 2016). A study showed that $C 7$ had increased expressed in liver cancer stem cells and enhanced the stemness of liver cancer cells by up-regulating Nanog, Oct4, Sox2, and C-myc (Seol et al. 2016). $C 7$ is also identified as a potential tumor suppressor and may serve as a prognostic biomarker for certain cancers (Chen et al. 2020; Ying et al. 2016). Complement gene polymorphism is closely related to the occurrence of cancer. DAF (decay accelerating factor), as one of key inhibitors of the complement system, inhibits complement activation by preventing the formation of $\mathrm{C} 3 / \mathrm{C} 5$ convertase from interfering with the formation of MAC (Mikesch et al. 2006; Spendlove et al. 2006). Studies have indicated that DAF rs $2564978 \mathrm{~T}>\mathrm{C}$ variant contributed to an increased risk of NSCLC (Zhang et al. 2017) and rs $10746463 \mathrm{G}>$ A polymorphism was related to elevated risk of gastric cancer (Song et al. 2015). Complement receptor $1(\mathrm{CR} 1)$, acting as the receptor of $\mathrm{C} 3 \mathrm{~b}$ and $\mathrm{C} 4 \mathrm{~b}$ to inhibit the complement activity (Liu \& Niu 2009). The tag genetic variant rs9429942 in CR1 had great effect on the susceptibility to gastric cancer (Zhao et al. 2015). However, the relationship between the polymorphisms of $C 7$ and the susceptibility to gastric cancer still needs to be explored.

In this study, we conducted a case-control study in the Chinese population to verify the hypothesis that the potential functional polymorphism of $C 7$ contributes to the susceptibility of gastric cancer.

MATERIALS AND METHODS

Peer] reviewing PDF | (2021:08:64943:1:1:NEW 22 Dec 2021) 


\section{$87 \quad$ Study population}

88 This case-control study contains 471 gastric cancer patients and 471 healthy controls. All

89

90

91

92

93

94

95

patients were recruited from North China University of Science and Technology Affiliated

Tangshan Renmin Hospital and Affiliated Tangshan Gongren Hospital from January 2011 to

May 2015. Healthy individuals were from a large population underwent physical examinations in same area during the same period. We also detected the $C 7$ expression level in serum of 70 healthy control and 70 gastric cancer patients. This study was approved by Institutional Review Board of North China University of Science and Technology (2019021). All subjects signed an informed consent form.

\section{Potentially functional SNPs filtering}

Based on NCBI dbSNP (https://www.ncbi.nlm.nih.gov/snp/) and Ensembl (http://asia.ensembl.org/index.html) database, we screened out all SNPs in the promoter (2000bp upstream of the transcription revelation site) and 3' untranslated region (3'UTR) of $C 7$ with minor allele frequency (MAF) over than 0.05 in Chinese population. Alibaba 2.1 (http://generegulation.com/pub/programs/alibaba2) tool was then used to predict the binding ability of transcription factors and SNP info Web server (https://manticore.niehs.nih.gov/) to predict the miRNA binding changes of SNPs in 3'UTR.

\section{Genotyping of Genetic Variants}

Peripheral blood DNA was extracted by using TIANamp Blood DNA Kit (TIANGEN, Beijing, China) according to manufacturer's instructions. PCR-restriction fragment length polymorphism (PCR-RFLP) analysis were applied for genotyping. The target DNA fragment containing C7 
108

109

110

111

112

113

114

115

116

117

118

119

120

121

122

123

124

125

126

127

rs1376178 or rs1061429 was amplified with primer pairs, rs1376178 $\mathrm{PF}\left(5^{\prime}\right.$ -

GCTAGAATCAATGCAAAGCTATGCG-3')/ PR (5'-TCAGATCACTGTGTTGGAAAGTT-

3') and rs1061429 PF (5'-GCTAGAATCAATGC AAAGCTATGCG-3')/ PR (5'-

AAGGAAAAGCTGTCCAGTGC-3'), respectively. PCR was performed in $6 \mu \mathrm{L}$ PCR reaction mixture with $1 \times$ Ftaq PCR Mix, 20 ng genomic DNA and $0.1 \mu \mathrm{M}$ each primer. The thermal cycling conditions for both $C 7 \mathrm{rs} 1376178$ and rs 1061429 variants were $3 \mathrm{~min}$ at $94^{\circ} \mathrm{C}$ followed by $30 \mathrm{~s}$ at $94^{\circ} \mathrm{C}, 30 \mathrm{~s}$ at $60^{\circ} \mathrm{C}$, and $30 \mathrm{~s}$ at $72^{\circ} \mathrm{C}$ for 30 cycles, and then a final extension 3 min at $72^{\circ} \mathrm{C}$. PCR products for $C 7 \mathrm{rs} 1376178 \mathrm{C}>\mathrm{A}(125 \mathrm{bp})$ and $C 7 \mathrm{rs} 1061429 \mathrm{C}>\mathrm{A}(242 \mathrm{bp})$ were digested by Hha I (NEB, Ipswich, MA, USA) and Nco I (NEB, Ipswich, MA, USA) and then was separated on 3\% agarose gel. For quality assurance, approximately $10 \%$ of the samples were randomly selected for re-genotyping and all results were in $100 \%$ concordance.

\section{Bioinformatic analysis of $C 7$ expression}

To analyze the levels of the $C 7$ mRNA expression in GC, the GEPIA databases were analyzed.

GEPIA (http://gepia.cancer-pku.cn/) is a comprehensive and interactive web resource for analyzing cancer data, which includes 9736 tumors and 8587 normal samples from Genotype-

Tissue Expression (GTEx) and The Cancer Genome Atlas (TCGA) (Tang et al. 2017).

Additionally, we also extracted eQTL data from the GTEx database, where differences in gene levels under different SNPs were examined. $P$-value with $<0.05$ was considered as statistical significance.

\section{Enzyme Linked Immunosorbent Assay (ELISA)}


128 The serum samples from 70 normal individuals and 70 gastric cancer patients were used to detect

129 the level of $C 7$ protein by using enzyme-linked immunosorbent assay (ELISA). Human

130 Complement (C7) kit were purchased from Cusabio company (Wuhan, China). We conducted

131 the $C 7$ expression analysis in serum according to manufactory's instructor.

\section{Cell culture}

133 Gastric cancer cell line (BGC823) was purchased from Cell Bank of Type Culture Collection of 134 the Chinese Academy of Sciences Shanghai Institute of Biochemistry and Cell Biology. BGC823

135 cells were cultured in RPMI-1640 (Thermo Fisher Scientific, Waltham, USA) supplemented with 10\% fetal bovine serum (FBS; Thermo Fisher Scientific, Waltham, USA) and 1\% antibiotics (100U/ml penicillin and $100 \mu \mathrm{g} / \mathrm{mL}$ streptomycin) in an atmosphere of 5\% CO2 at $13837^{\circ} \mathrm{C}$.

Plasmid constructure and luciferase reporter gene assay

A $1615 \mathrm{bp}$ DNA fragment containing rs1376178 site in the promoter of $C 7$ were inserted into

pGL3-basic plasmid (Promega, Madison, USA) to conduct luciferase reporter gene assay. The

PCR primers with Kpn I and Xho I (NEB, Ipswich, USA) cutting site adaptor were 5'-

GGGTACCCTTTCCCACTTCCAGTGGTGC-3') and 5'-CCGCTCGAG

CTGAGATTTAGCTCCTACCCC-3'. The final plasmids with rs1376178 C or A allele were designed as pGL3 $3_{\mathrm{rs} 1376178 \mathrm{C}}$ and pGL3 ${ }_{\mathrm{rs} 1376178 \mathrm{~A}}$, respectively. A $1268 \mathrm{bp}$ fragment with rs1061429 site in the 3' untranslated region (UTR) was cloned into psiCHECK2 plasmid (Promega, Madison, USA). The PCR primer pairs with Xho I and Not I (NEB, Ipswich, USA) sites were 5'- 
149 CTGGGACTGTATCCACAGAA-3'. The constructors with rs1061429C or A allele were named

150 as psiCHECK $2_{\text {rs } 1061429 \mathrm{C}}$ and $\mathrm{psiCHECK} 2_{\mathrm{rs} 1061429 \mathrm{~A}}$, respectively.

151 BGC823 gastric cancer cells were seeded in 24-well plates at a density of $2 \times 10^{5}$ cells/well. As

152 the cells reached 60-70\% confluent, 300ng of each pGL3-containing plasmids and 5ng pRL-

153 SV40 (Promega, Madison, USA) were co-transfected into the cells using Lipofectamine 2000

154 (Invitrogen, Carlsbad, USA). For psiCHECK2-containing plasmids, 20ng of plasmids was

155 transfected into gastric cancer cells with or without 30pmol of miR-591 mimic (GenePharma,

156 Shanghai, China). Gastric cancer cells were then collected $24 \mathrm{~h}$ after transfection. The activity of

157 luciferase and renilla reporter gene activity were measured using GloMax 20/20 Luminometer

158 (Promega, Madison, USA).

159 Statistical analysis

160 The statistical analyses in our study were conducted by SPSS 23.0 (SPSS Inc., Chicago, USA).

161 The Hardy-Weinberg equilibrium (HWE) of $C 7$ polymorphisms in controls were assessed by $\chi^{2}$

162 test. Differences of basic characteristics between cases and control subjects were evaluated by $\chi^{2}$

163 test. The association of $C 7$ genotypes with the susceptibility to gastric cancer was evaluated by

164 unconditional logistic regression with OR (95\% CI) after adjusted by age, sex, smoking status

165 and drinking status. The interaction between gene and environment was analyzed by epiR

166 program in R platform (version 3.6.1). We defined the smokers as they smoked more than 100

167 cigarettes in their lifetime. Drinkers were categorized as the individuals took at least 12 drinks on

168 one occasion during the previous year according to international guide for monitoring alcohol

169 consumption and harm of WHO. $P$ value $<0.05$ was regarded as statistical significance. To

Peer] reviewing PDF | (2021:08:64943:1:1:NEW 22 Dec 2021) 
170

171

172

173

174

175

176

177

178

179

180

181

182

183

184

185

186

187

188

189

190

explore the associations of $C 7$ expression level and the prognosis of gastric cancer, we

performed survival analysis by Kaplan-Meier online program (www.kmpolt.com).

\section{RESULTS}

\section{Subject Characteristics.}

The distribution of select characteristics of all subjects were shown in Table 1. The study involved in 471 gastric cancer patients and 471 healthy controls. There was no statistically significant difference in distribution of age and gender between cases and controls (age: $P=$ 1.00; gender $P=0.44$ ). Regard to the distribution of smoking and drinking status, there was no significant difference between cases and controls (smoking: $P=0.32$; drinking $P=0.25$ ).

\section{Association of $C 7$ gene polymorphisms with gastric cancer risk}

After predicting potential regulatory functional SNPs, we found that $C 7 \mathrm{rs} 1376178 \mathrm{C}>\mathrm{A}$ variant enhanced the binding capability to transcription factor STAT1 and C7 rs1061429 C $>$ A allele created a binding site with has-miR-591 in 3'UTR. Genotype distributions of rs 1376178 and rs 1061429 polymorphisms in controls were conformed to Hardy-Weinberg equilibrium (HWE) (Table2).

The relationship between each genetic variant and the susceptibility to gastric cancer was shown in Table 3. After adjusted by gender, age, drinking and smoking status, non-conditional logistic regression analysis showed that the distribution of $C 7$ rs1376178 AA and CA genotypes was statistically different between cases and controls $(P<0.001)$. The individuals with $C 7$ rs 1376178

AA or CA genotype had a higher risk of GC with OR (95\%CI) of 2.09 (1.43-3.03) and 
191

192

193

194

195

196

197

198

199

200

201

202

203

204

205

206

207

208

209

210

211

1.88(1.35-2.63), respectively. For $C 7$ rs1061429 C >A polymorphism, AA genotype was

associated with the elevated risk for developing gastric cancer $(\mathrm{OR}=2.16,95 \% C I=1.37-3.38)$.

\section{Stratification analysis of $C 7$ variants with GC risk}

To further analyze the effect of age, gender, smoking and drinking on the association of $C 7$

variants (rs 1376178 and rs 1061429) with the risk of GC, we performed stratification analysis

(Table 4). Gender stratification analysis showed that $C 7$ rs 1376178 AA genotype was associated

with the elevated risk of gastric cancer among males $(\mathrm{OR}=2.88,95 \% C I=1.81-4.58, P<$

$0.001)$, but not among females $(\mathrm{OR}=1.06,95 \% C I=0.55-2.06, P>0.05)$. When stratified by

age, individuals with $C 7$ rs1376178 AA had an increased risk of gastric cancer in both groups

with OR $(95 \% C I)$ of $2.19(1.26-3.78)$ for the youngers and $2.07(1.23-3.48)$ for the elders. Our

data also showed that the risk of gastric cancer was associated with the rs1376178 AA regardless

of smoking and drinking status $(\mathrm{OR}=2.05,95 \% C I=1.31-3.21, P=0.002$ for nonsmokers; OR

$=2.40,95 \% C I=1.19-4.87, P=0.015$ for smokers; $\mathrm{OR}=1.94,95 \% C I=1.28-2.93, P=0.002$

for nondrinkers; $\mathrm{OR}=3.55,95 \% C I=1.37-9.17, P=0.009$ for drinkers).

Stratification analysis of $C 7$ rs1061429 polymorphism was showed in Table5. Our data

suggested that individuals with rs1061429 AA had an increased risk of gastric cancer among youngers $(\mathrm{OR}=2.84,95 \% C I=1.39-5.80, P=0.004)$ and non-smokers $(\mathrm{OR}=2.79,95 \% C I=$

1.63-4.77, $P<0.001)$, but not among elders $(\mathrm{OR}=1.81,95 \% C I=1.00-3.28, P=0.050)$ and

smokers $(\mathrm{OR}=1.03,95 \% C I=0.43-2.5, P=0.947)$. The stratification analysis by gender or

drinking status showed that rs1061429 AA genotype was contributed to the risk of gastric cancer

regardless of gender and drinking status $(\mathrm{OR}=1.94,95 \% C I=1.13-3.33, P=0.017$ for males; 
$212 \mathrm{OR}=2.87,95 \% C I=1.26-6.54, P=0.012$ for females; $\mathrm{OR}=1.92,95 \% C I=1.18-3.13, P=$

2130.008 for nondrinkers; $\mathrm{OR}=5.23,95 \% C I=1.34-20.40, P=0.017$ for drinkers). Use $\mathrm{R}$ package

214 epiR to build Logistic regression gene-environment interaction mode, we found that rs 1061429

215 had no additive and multiplicative interaction with drinking or gender to affect the risk of gastric

216 cancer $(P>0.05)$.

217 The expression of $C 7$ in gastric cancer and its effect on prognosis and clinical-pathological

218 characteristics of gastric cancer

219 Furthermore, we explore the potential function of gene $C 7$ in gastric cancer, based on GEPIA

data, we analyzed the differential expression of $C 7$ in gastric cancer tissues and adjacent normal

tissues and found that the level of $C 7$ mRNA in gastric cancer tissues $(n=408)$ was significantly

depressed when compared with that in adjacent normal tissues $(n=211)$ (Fig. 1A). To verify the

result from GEPIA data, we further compared the expression of $C 7$ in serum between normal

individuals and gastric cancer patients and found a significant differential expression of $C 7(P=$

0.043) (Fig. 1B).

We also analyzed the impact of $C 7$ expression on the prognosis of gastric cancer using Kaplan-

Meier online program and demonstrated that higher expression of $C 7$ was related with poor

overall survival time (OS) and post-progression survival time (PPS) of gastric patients with HR

$(95 \% C I)$ of $1.29(1.09-1.53)$ and $1.51(1.19-1.91)$, respectively (Fig.1C and 1D). We also

evaluated the association of $C 7$ expression level with TNM stage. Our data didn't show any

correlation between $C 7$ expression and TNM stage (Supplementary Figure 1).

\section{$C 7$ genotypes and clinical-pathological characteristics}


233 We used Kendall's rank correlation tests to estimate the correlation between these two SNPs and

234 clinicopathological characteristics. Our results showed that rs1061029 C $>$ A variant has a positive

235 correlation with lymph node metastasis, but the correlation strength was low $(P=0.022$,

236 Kendall's Tau- $b=0.104)$. We didn't find any correlation between rs $1376178 \mathrm{C}>\mathrm{A}$ variant and

237 TNM stage, tumor size, lymph node metastasis, distant metastasis (Table 6).

238 The effect of $C 7$ polymorphisms on the regulatory activity of $C 7$

239 To substantiate the association between the identified SNPs and GC risk, using the genotype and 240 gene expression data of 324 normal gastric tissues in the GTEx to analyze, the eQTL data results 241 showed that the mRNA expression of $C 7$ was significantly related to the rs 1061429 genotype $(P$ $242<0.001)$ (Fig. 2A). However, there is no eQTL data related to rs1376178 in GEPIA database.

243 We then measured the $C 7$ protein level in serum of 70 individuals. For rs 1061429 variant, the expression of $C 7$ was significantly lower in individuals with AA genotype when compared with those with CC genotype $(P=0.029)$ (Fig. 2B). For rs1376178 polymorphism, our data didn't show any effect on $C 7$ expression (Fig. 2C).

247 Our luciferase reporter assay showed that $\mathrm{pGL} 3_{\mathrm{rs} 1376178 \mathrm{C}}$ exhibited 2.86-fold higher luciferase activity than pGL $3_{\mathrm{rs} 1376178 \mathrm{~A}}(P<0.001)\left(\right.$ Fig. 3A). We also found that psiCHECK $2_{\mathrm{rs} 1061429 \mathrm{~A}}$ had 1.34-fold increased luciferase activity in comparison to psiCHECK $2_{\mathrm{rs} 1061429 \mathrm{C}}$ when co-transfected with miR-591 $(P=0.0012)$ (Fig. 3B). There was no effect of rs1061429 polymorphism on the reporter gene activity without additional miR-591.

\section{DISCUSSION}


254 The development of gastric cancer is a long-term multistage process with complex etiology.

255 Genetic epidemiological studies have shown that gastric cancer is the result of long-term effects

256 of environmental and individual genetic factors (Jin et al. 2020). Many studies provided strong

257 evidence for the effect of complement activation on tumor development (Kwak et al. 2018;

258 Markiewski et al. 2008; Roumenina et al. 2019). C7 is a terminal component of complement

259 activation which plays essential roles within innate immunity (Fujita et al. 2004; Walport 2001).

260 Membrane-associated $C 7$ was acted a regulator of the excessive proinflammatory reaction (Bossi

261 et al. 2009). As an essential part of the membrane attack complex (MAC), $C 7$ participated in

262 various microbial defense responses and immune injury responses. The lack of $C 7$ may affect the

263 function of MAC and further increase the susceptibility to infection (Barroso et al. 2010; Sarma

264 \& Ward 2011). Tumor infection promoted cancer aggression, and prevented inflammation

265 reduces metastasis and improved anti-tumor treatment (Maller et al. 2021).C7 played an

266 important role in the occurrence of various cancers. It was demonstrated that $C 7$ affected the

267 progression of liver cancer via affecting the transcription of stemness factors (Seol et al. 2016).

268 Similarly, $C 7$ was verified to be related to the prognosis of prostate cancer patients (Chen et al.

269 2020). Consistent with our finding which showed a significantly down-regulated $C 7$ in gastric

270 cancer tissue, researchers also found that the $C 7$ was down expressed in ovarian, it was also

271 demonstrated that the decreased expression of $C 7$ was related to poor differentiation in patients

272 with NSCLC (Ying et al. 2016). These studies indicated its significance in the occurrence and

273 development of tumors.

274 Single Nucleotide Polymorphism (SNP) was widely present in human genome and is the most 
275

276

277

278

279

280

281

282

283

284

285

286

287

288

289

290

291

292

293

294

295

common type of genetic variation, which can affect gene regulation by changing gene structure or expression (Zhang et al. 2014). There are few reports on the relationship between $C 7$ genetic variants and cancer risk. In this study, we discovered that $C 7 \mathrm{rs} 1376178 \mathrm{C}>\mathrm{A}$ increased the susceptibility to gastric cancer. This was consistent with the $C 7$ expression analysis and dualluciferase reporter gene results which showed that the rs1376178 A allele significantly reduced the promoter activity and the expression level of $C 7$. For rs1061429 polymorphism, we found that $C 7 \mathrm{rs} 1061429 \mathrm{C}>\mathrm{A}$ increased the binding ability of has-miR-591 to reduce the activity of reporter gene. Literature reported dysregulation of miR-591 confer paclitaxel resistance to ovarian cancer (Huh et al. 2013), and circ_0091581 could promote the progression of hepatocellular carcinoma through miR-591/FOSL2 Axis (Ji et al. 2020). Therefore, binding of hsa-mir-591 may inhibit translation of $C 7$ and attenuate its expression further participate in the development of gastric cancer.

Gastric cancer is a complex disease, in which the interaction of genetic variants with several compounding factors, such as age, sex and environment, has been demonstrated to modulate the risk phenotypes (Favé et al. 2018; Jiang et al. 2021). For gastric cancer, the environmental risk factors involved in Helicobacter pylori (H. pylori) infection, smoking and drinking (Brenner et al. 2009; Terry et al. 2002). Due to the missing data of H. pylori infection, we only analyzed the impact of these compounding factors, including age, sex, smoking status and drinking status on genetic risk of disease.

Smoking was one of established and important risk factors contributing to the risk of gastric cancer (Butt et al. 2019). Thus, we analyzed the effects of $C 7$ variants on the risk of GC when 
296

297

298

299

300

301

302

303

304

305

306

307

308

309

310

311

312

313

314

315

316

stratified by smoking status. Our data presented that rs1061429 variant had an effected on gastric cancer risk among non-smokers, but not among smokers. Cigarette smoke contains multiple

known human carcinogens. Exposure to nicotine-derived nitrosamine ketone, a key carcinogenic ingredient of cigarette smoke, had been proved to result in mitochondrial dysfunction (Wu et al. 2019), to promote immune dysfunction, and further to influence tumor immune microenvironment (de la Iglesia et al. 2020; Lee et al. 2012). It has been reported that cigarette smoke can induce oxidative injury and dose-dependently stimulated gastric cancer cell proliferation (Bhattacharyya et al. 2014; Shin et al. 2004). Smoking status could affect the association of $C 7$ rs1061429 variant with the gastric cancer risk which was consistent with the report on gene-environment interaction between smoking and SNP in decay-accelerating factor (DAF) gene (Song et al. 2015). This could be supported by which tobacco enhanced the activation of the classical pathway of the complement system (Yin et al. 2008).

Drinking was another important risk factor for gastric cancer (Ma et al. 2017; Na \& Lee 2017). In current study, individuals with $C 7$ rs1376178AA or rs 1061429AA genotype were contributed to the risk of gastric cancer regardless of drinking status. The result was consistent with some previous studies that drinking significantly effect on association between polymorphisms and gastric cancer risk (Li et al. 2021; Qiu et al. 2015).

Besides smoking and drinking, age also contributed to the occurrence and development of a variety of cancers (Hansen et al. 2019). In this study, when stratified by age, our data showed that individuals carrying $C 7$ rs1061429 AA genotype had an increased risk of gastric cancer among youngers, but not among elders. The pathogenesis of gastric cancer in the elders was 
317 mostly induced by environmental factors (Forman \& Burley 2006); however, young patients are

318 more effected by genetic factors (Machlowska et al. 2020). Several previous studies from our

319 laboratory also provided the evidence that the effect of genetic variants in TNFSF15 and XAB2

320 on the susceptibility to gastric cancer could be modified by age (Gao et al. 2019; Pei et al. 2015).

321 These findings further verified the importance of interaction of age with the genetic

322 polymorphism on the development of gastric cancer.

323 Gender was also an important factor affecting the development of cancers (Li et al. 2019; Lou et

al. 2020). When stratified by gender, our data shows that individuals with the $C 7$ rs1061429 AA

genotype have an increased risk of gastric cancer in both men and women, but the risk in women

is significantly higher than that in men. This might be related to different lifestyle between men

and women (Song et al. 2008) and other risk factors, such as diet, microbial virulence, and Hp

infection (Cover \& Peek 2013; Xia et al. 2016). Similarly, researchers found that $C 7$ rs1063499

GG genotype increased the risk of liver cancer among men, but not among women (de Lima et

al. 2018). Our previous study demonstrated that TNFSF15 -638 GG genotype was associated

with an increased risk of SCLC among males compared with the AA genotype, but not among

females (Gao et al. 2019). These findings further indicated the contribution of gender to cancer

development.

There are still some limitations. The sample size is relatively small after stratification. In

addition, as one of the best-established environmental factors of gastric cancer, $\mathrm{H}$. Pylori 
compounding factors.

339

340 CONCLUSIONS

341 In summary, our study demonstrated that $C 7$ genetic variants were related to gastric cancer's

342 susceptibility which implying the critical role of the complement genes in the development of

343 gastric cancer.

\section{LIST OF ABBREVIATIONS}

C7: Complement component 7

347 GC: Gastric cancer

GEO: Gene Expression Omnibus

PCR-RFLP: Polymerase chain reaction-restriction fragment length polymorphism technique

MAC: Membrane attack complex

HWE: Hardy-Weinberg equilibrium

\section{ADDITIONAL INFORMATION AND DECLARATIONS}

\section{Funding}

This study was funded by Foundation of Key Project of Natural Science Foundation of Hebei

province of China (H2017209233 to X. Zhang), Leader talent cultivation plan of innovation team in Hebei province (No. LJRC001). The funders were not involved in the design of the study, collection, analysis, interpretation of data and writing the manuscript. 


\section{Grant Disclosures}

361 The following grant information was disclosed by the authors:

362 Natural Science Foundation of Hebei province of China: H2017209233.

363 Leader talent cultivation plan of innovation team in Hebei province: No. LJRC001.

365 Competing Interests

366 The authors declare there are no competing interests.

\section{Author Contributions}

369

370

371

372

373

374 
380 The raw measurements are available in Tables 1-5. Anonymized patient information is 381 available in Supplemental Files.

382

383

384

385

386

387

388

389

390

391

392

393

394

395

396

397

398

399

400

\section{REFERENCES}

Bao D, Zhang C, Li L, Wang H, Li Q, Ni L, Lin Y, Huang R, Yang Z, Zhang Y, and Hu Y. 2020. Integrative Analysis of Complement System to Prognosis and Immune Infiltrating in Colon Cancer and Gastric Cancer. Front Oncol 10:553297. 10.3389/fonc.2020.553297

Barroso S, López-Trascasa M, Merino D, Alvarez AJ, Núñez-Roldán A, and Sánchez B. 2010. C7 deficiency and meningococcal infection susceptibility in two spanish families. Scand J Immunol 72:38-43. 10.1111/j.1365-3083.2010.02403.x

Berraondo P, Minute L, Ajona D, Corrales L, Melero I, and Pio R. 2016. Innate immune mediators in cancer: between defense and resistance. Immunol Rev 274:290-306. 10.1111/imr.12464

Bhattacharyya A, Chattopadhyay R, Mitra S, and Crowe SE. 2014. Oxidative stress: an essential factor in the pathogenesis of gastrointestinal mucosal diseases. Physiol Rev 94:329-354. 10.1152/physrev.00040.2012

Bossi F, Rizzi L, Bulla R, Debeus A, Tripodo C, Picotti P, Betto E, Macor P, Pucillo C, Würzner R, and Tedesco F. 2009. C7 is expressed on endothelial cells as a trap for the assembling terminal complement complex and may exert anti-inflammatory function. Blood 113:3640-3648. 10.1182/blood-2008-03-146472

Brenner H, Rothenbacher D, and Arndt V. 2009. Epidemiology of stomach cancer. Methods Mol 
401

402

403

404

405

406

407

408

409

410

411

412

413

414

415

416

417

418

419

420

421

Biol 472:467-477. 10.1007/978-1-60327-492-0_23

Butt J, Varga MG, Wang T, Tsugane S, Shimazu T, Zheng W, Abnet CC, Yoo KY, Park SK, Kim J, Jee SH, Qiao YL, Shu XO, Waterboer T, Pawlita M, and Epplein M. 2019. Smoking, Helicobacter Pylori Serology, and Gastric Cancer Risk in Prospective Studies from China, Japan, and Korea. Cancer Prev Res (Phila) 12:667-674. 10.1158/19406207.Capr-19-0238

Chen W, Zheng R, Baade PD, Zhang S, Zeng H, Bray F, Jemal A, Yu XQ, and He J. 2016. Cancer statistics in China, 2015. CA Cancer J Clin 66:115-132. 10.3322/caac.21338

Chen Z, Yan X, Du GW, Tuoheti K, Bai XJ, Wu HH, Zhang RJ, Xiao GF, and Liu TZ. 2020. Complement C7 (C7), a Potential Tumor Suppressor, Is an Immune-Related Prognostic Biomarker in Prostate Cancer (PC). Front Oncol 10:1532. 10.3389/fonc.2020.01532

Cover TL, and Peek RM, Jr. 2013. Diet, microbial virulence, and Helicobacter pylori-induced gastric cancer. Gut Microbes 4:482-493. 10.4161/gmic. 26262

de la Iglesia JV, Slebos RJC, Martin-Gomez L, Wang X, Teer JK, Tan AC, Gerke TA, AdenBuie G, van Veen T, Masannat J, Chaudhary R, Song F, Fournier M, Siegel EM, Schabath MB, Wadsworth JT, Caudell J, Harrison L, Wenig BM, Conejo-Garcia J, Hernandez-Prera JC, and Chung CH. 2020. Effects of Tobacco Smoking on the Tumor Immune Microenvironment in Head and Neck Squamous Cell Carcinoma. Clin Cancer Res 26:1474-1485. 10.1158/1078-0432.Ccr-19-1769

de Lima RE, de Holanda Martins CM, do Carmo RF, Aroucha D, Pereira L, Vasconcelos LRS, and Moura P. 2018. Two sides of a coin: GG genotype of C7 provides protection against 
422

423

424

425

426

427

428

429

430

431

432

433

434

435

436

437

438

439

440

441

442

fibrosis severity while showing a higher risk for hepatocellular carcinoma in patients with hepatitis C. Hum Immunol 79:702-707. 10.1016/j.humimm.2018.06.009

Digklia A, and Wagner AD. 2016. Advanced gastric cancer: Current treatment landscape and future perspectives. World J Gastroenterol 22:2403-2414. 10.3748/wjg.v22.i8.2403

Favé MJ, Lamaze FC, Soave D, Hodgkinson A, Gauvin H, Bruat V, Grenier JC, Gbeha E, Skead K, Smargiassi A, Johnson M, Idaghdour Y, and Awadalla P. 2018. Gene-by-environment interactions in urban populations modulate risk phenotypes. Nat Commun 9:827. 10.1038/s41467-018-03202-2

Forman D, and Burley VJ. 2006. Gastric cancer: global pattern of the disease and an overview of environmental risk factors. Best Pract Res Clin Gastroenterol 20:633-649. 10.1016/j.bpg.2006.04.008

Fujita T, Matsushita M, and Endo Y. 2004. The lectin-complement pathway--its role in innate immunity and evolution. Immunol Rev 198:185-202. 10.1111/j.0105-2896.2004.0123.x

Gao H, Niu Z, Zhang Z, Wu H, Xie Y, Yang Z, Li A, Jia Z, and Zhang X. 2019. TNFSF15 promoter polymorphisms increase the susceptibility to small cell lung cancer: a casecontrol study. BMC Med Genet 20:29. 10.1186/s12881-019-0762-6

Hansen MB, Ross L, Petersen MA, and Groenvold M. 2019. Age, cancer site and gender associations with symptoms and problems in specialised palliative care: a large, nationwide, register-based study. BMJ Support Palliat Care. 10.1136/bmjspcare-2019001880

Huh JH, Kim TH, Kim K, Song JA, Jung YJ, Jeong JY, Lee MJ, Kim YK, Lee DH, and An HJ. 
2013. Dysregulation of miR-106a and miR-591 confers paclitaxel resistance to ovarian cancer. Br J Cancer 109:452-461. 10.1038/bjc.2013.305

445 Ji C, Hong X, Lan B, Lin Y, He Y, Chen J, Liu X, Ye W, Mo Z, She Z, and Lin S. 2020. Circ_0091581 Promotes the Progression of Hepatocellular Carcinoma Through Targeting miR-591/FOSL2 Axis. Dig Dis Sci. 10.1007/s10620-020-06641-4

448 449 450 451

454 455 456 457 458 459 460

Jiang X, Holmes C, and McVean G. 2021. The impact of age on genetic risk for common diseases. PLoS Genet 17:e1009723. 10.1371/journal.pgen.1009723

Jin G, Lv J, Yang M, Wang M, Zhu M, Wang T, Yan C, Yu C, Ding Y, Li G, Ren C, Ni J, Zhang R, Guo Y, Bian Z, Zheng Y, Zhang N, Jiang Y, Chen J, Wang Y, Xu D, Zheng H, Yang L, Chen Y, Walters R, Millwood IY, Dai J, Ma H, Chen K, Chen Z, Hu Z, Wei Q, Shen H, and Li L. 2020. Genetic risk, incident gastric cancer, and healthy lifestyle: a metaanalysis of genome-wide association studies and prospective cohort study. Lancet Oncol $21: 1378-1386.10 .1016 / \mathrm{s} 1470-2045(20) 30460-5$

Kwak JW, Laskowski J, Li HY, McSharry MV, Sippel TR, Bullock BL, Johnson AM, Poczobutt JM, Neuwelt AJ, Malkoski SP, Weiser-Evans MC, Lambris JD, Clambey ET, Thurman JM, and Nemenoff RA. 2018. Complement Activation via a C3a Receptor Pathway Alters CD4(+) T Lymphocytes and Mediates Lung Cancer Progression. Cancer Res 78:143-156. 10.1158/0008-5472.Can-17-0240

Lee J, Taneja V, and Vassallo R. 2012. Cigarette smoking and inflammation: cellular and molecular mechanisms. J Dent Res 91:142-149. 10.1177/0022034511421200

Li H, Wang C, Wei Z, Chen W, Guo Z, He Y, and Zhang C. 2019. Differences in the prognosis 
464

465

466

467

468

469

470

471

472

473

474

475

476

477

478

479

480

481

482

483

484

of gastric cancer patients of different sexes and races and the molecular mechanisms involved. Int J Oncol 55:1049-1068. 10.3892/ijo.2019.4885

Li Z, Gao H, Liu Y, Wu H, Li W, Xing Y, Zhang Z, and Zhang X. 2021. Genetic variants in the regulation region of TLR4 reduce the gastric cancer susceptibility. Gene 767:145181. 10.1016/j.gene.2020.145181

Liu D, and Niu ZX. 2009. The structure, genetic polymorphisms, expression and biological functions of complement receptor type 1 (CR1/CD35). Immunopharmacol Immunotoxicol 31:524-535. 10.3109/08923970902845768

Lou L, Wang L, Zhang Y, Chen G, Lin L, Jin X, Huang Y, and Chen J. 2020. Sex difference in incidence of gastric cancer: an international comparative study based on the Global Burden of Disease Study 2017. BMJ Open 10:e033323. 10.1136/bmjopen-2019-033323

Ma K, Baloch Z, He TT, and Xia X. 2017. Alcohol Consumption and Gastric Cancer Risk: A Meta-Analysis. Med Sci Monit 23:238-246. 10.12659/msm.899423

Machlowska J, Baj J, Sitarz M, Maciejewski R, and Sitarz R. 2020. Gastric Cancer: Epidemiology, Risk Factors, Classification, Genomic Characteristics and Treatment Strategies. Int J Mol Sci 21. 10.3390/ijms21114012

Maller O, Drain AP, Barrett AS, Borgquist S, Ruffell B, Zakharevich I, Pham TT, Gruosso T, Kuasne H, Lakins JN, Acerbi I, Barnes JM, Nemkov T, Chauhan A, Gruenberg J, Nasir A, Bjarnadottir O, Werb Z, Kabos P, Chen YY, Hwang ES, Park M, Coussens LM, Nelson AC, Hansen KC, and Weaver VM. 2021. Tumour-associated macrophages drive stromal cell-dependent collagen crosslinking and stiffening to promote breast cancer 

aggression. Nat Mater 20:548-559. 10.1038/s41563-020-00849-5

486

487

488

489

490

491

492

493

494

495

496

497

498

499

500

501

502

503

504

505

Markiewski MM, DeAngelis RA, Benencia F, Ricklin-Lichtsteiner SK, Koutoulaki A, Gerard C, Coukos G, and Lambris JD. 2008. Modulation of the antitumor immune response by complement. Nat Immunol 9:1225-1235. 10.1038/ni.1655

Mikesch JH, Schier K, Roetger A, Simon R, Buerger H, and Brandt B. 2006. The expression and action of decay-accelerating factor (CD55) in human malignancies and cancer therapy. Cell Oncol 28:223-232. 10.1155/2006/814816

Na HK, and Lee JY. 2017. Molecular Basis of Alcohol-Related Gastric and Colon Cancer. Int J Mol Sci 18. 10.3390/ijms18061116

Ostrand-Rosenberg S. 2008. Cancer and complement. Nat Biotechnol 26:1348-1349. $10.1038 / \mathrm{nbt} 1208-1348$

Park SK, Yang JJ, Oh S, Cho LY, Ma SH, Shin A, Ko KP, Park T, Yoo KY, and Kang D. 2012. Innate immunity and non-Hodgkin's lymphoma (NHL) related genes in a nested casecontrol study for gastric cancer risk. PLoS One 7:e45274. 10.1371/journal.pone.0045274

Pei N, Cao L, Liu Y, Wu J, Song Q, Zhang Z, Yuan J, and Zhang X. 2015. XAB2 tagSNPs contribute to non-small cell lung cancer susceptibility in Chinese population. $B M C$ Cancer 15:560. 10.1186/s12885-015-1567-4

Qiu LX, He J, Cheng L, Zhou F, Wang MY, Sun MH, Zhou XY, Li J, Guo WJ, Wang YN, Yang YJ, Wang JC, Jin L, Zhu XD, and Wei QY. 2015. Genetic variant of PRKAA1 and gastric cancer risk in an eastern Chinese population. Oncotarget 6:42661-42666. 
506 Roumenina LT, Daugan MV, Noé R, Petitprez F, Vano YA, Sanchez-Salas R, Becht E,

507

508

509

510

511

512

513

514

515

516

517

518

519

520

521

522

523

524

525

526

Meilleroux J, Clec'h BL, Giraldo NA, Merle NS, Sun CM, Verkarre V, Validire P, Selves

J, Lacroix L, Delfour O, Vandenberghe I, Thuilliez C, Keddani S, Sakhi IB, Barret E,

Ferré P, Corvaïa N, Passioukov A, Chetaille E, Botto M, de Reynies A, Oudard SM,

Mejean A, Cathelineau X, Sautès-Fridman C, and Fridman WH. 2019. Tumor Cells

Hijack Macrophage-Produced Complement C1q to Promote Tumor Growth. Cancer

Immunol Res 7:1091-1105. 10.1158/2326-6066.Cir-18-0891

Saeki N, Ono H, Sakamoto H, and Yoshida T. 2013. Genetic factors related to gastric cancer susceptibility identified using a genome-wide association study. Cancer Sci 104:1-8. $10.1111 /$ cas. 12042

Sarma JV, and Ward PA. 2011. The complement system. Cell Tissue Res 343:227-235.

10.1007/s00441-010-1034-0

Seol HS, Lee SE, Song JS, Rhee JK, Singh SR, Chang S, and Jang SJ. 2016. Complement proteins C7 and CFH control the stemness of liver cancer cells via LSF-1. Cancer Lett 372:24-35. 10.1016/j.canlet.2015.12.005

Shin VY, Liu ES, Ye YN, Koo MW, Chu KM, and Cho CH. 2004. A mechanistic study of cigarette smoke and cyclooxygenase- 2 on proliferation of gastric cancer cells. Toxicol Appl Pharmacol 195:103-112. 10.1016/j.taap.2003.10.009

Siegel RL, Miller KD, Fuchs HE, and Jemal A. 2021. Cancer Statistics, 2021. CA Cancer J Clin 71:7-33. 10.3322/caac.21654

Song HJ, Kim HJ, Choi NK, Hahn S, Cho YJ, and Park BJ. 2008. Gender differences in gastric 
cancer incidence in elderly former drinkers. Alcohol 42:363-368.

Song Q, Zhang Z, Liu Y, Han S, and Zhang X. 2015. The tag SNP rs10746463 in decayaccelerating factor is associated with the susceptibility to gastric cancer. Mol Immunol 63:473-478. 10.1016/j.molimm.2014.10.006

Song Z, Wu Y, Yang J, Yang D, and Fang X. 2017. Progress in the treatment of advanced gastric cancer. Tumour Biol 39:1010428317714626. 10.1177/1010428317714626

Spendlove I, Ramage JM, Bradley R, Harris C, and Durrant LG. 2006. Complement decay accelerating factor (DAF)/CD55 in cancer. Cancer Immunol Immunother 55:987-995. $10.1007 / \mathrm{s} 00262-006-0136-8$

Tang Z, Li C, Kang B, Gao G, Li C, and Zhang Z. 2017. GEPIA: a web server for cancer and normal gene expression profiling and interactive analyses. Nucleic Acids Res 45:W98w102. 10.1093/nar/gkx247

Terry MB, Gaudet MM, and Gammon MD. 2002. The epidemiology of gastric cancer. Semin Radiat Oncol 12:111-127. 10.1053/srao.30814

Walport MJ. 2001. Complement. First of two parts. N Engl J Med 344:1058-1066.

$$
\text { 10.1056/nejm200104053441406 }
$$

Wang F, Meng W, Wang B, and Qiao L. 2014. Helicobacter pylori-induced gastric inflammation and gastric cancer. Cancer Lett 345:196-202. 10.1016/j.canlet.2013.08.016

Wu S, Li X, Meng S, Fung T, Chan AT, Liang G, Giovannucci E, De Vivo I, Lee JH, and Nan H. 2019. Fruit and vegetable consumption, cigarette smoke, and leukocyte mitochondrial 
Würzner R. 2000. Modulation of complement membrane attack by local C7 synthesis. Clin Exp

Xia ZG, Yin HF, Long Y, Cheng L, Yu LJ, Guo WJ, Zhu XD, Li J, Wang YN, Yang YJ, Wang JC, Jin L, Qiu LX, and Wei Y. 2016. Genetic variant of miR-146a rs2910164 C>G and gastric cancer susceptibility. Oncotarget 7:34316-34321. 10.18632/oncotarget.8814 Thromb Res 122:221-228. 10.1016/j.thromres.2007.11.005 
570 Fig. $1 C 7$ expression in gastric cancer and its effect on the prognosis of gastric cancer patients

571 (A) $C 7$ expression in gastric cancer and adjacent normal tissues in GEPIA database. $(* P<0.05)$.

572 (B) $C 7$ expression in serum of normal individuals and gastric cancer patient. (C) Overall survival 573 and (D) post-progression survival analysis of gastric cancer patients based on $C 7$ expression.

Fig. 2 Association of $C 7$ expression in gastric cancer patients with $C 7$ genetic polymorphisms

576

577

578

579

580

581

(A) rs1061429 genotypes from GTEx database. (B) rs1061429 genotypes and (C) rs1376178 genotypes in serum.

Fig 3. The effect of $C 7$ polymorphisms on transcription activity. (A) Different allele of rs 1376178 had different regulatory effects in BGC823 cells. (B) Different allele of rs1061429 had different 3'UTR activity in BGC823 cells. ${ }^{* * *} P<0.001 ; * * P<0.01$. 


\section{Table $\mathbf{1}$ (on next page)}

Frequency distribution of the study population. 
1 Table 1 Frequency distribution of the study population.

\begin{tabular}{|c|c|c|c|c|c|}
\hline \multirow{2}{*}{ Variables } & \multicolumn{2}{|c|}{ Cases $(n=471)$} & \multicolumn{2}{|c|}{ Controls $(n=471)$} & \multirow{2}{*}{$P$ value } \\
\hline & NO & $\%$ & NO & $\%$ & \\
\hline \multicolumn{6}{|l|}{ Gender } \\
\hline Male & 332 & 70.5 & 321 & 68.2 & 0.44 \\
\hline Female & 139 & 29.5 & 150 & 31.8 & \\
\hline \multicolumn{6}{|l|}{ Age } \\
\hline$<60$ & 209 & 44.4 & 209 & 44.4 & 1.00 \\
\hline$\geq 60$ & 262 & 55.6 & 262 & 55.6 & \\
\hline \multicolumn{6}{|c|}{ Smoking status } \\
\hline Non-smoker & 323 & 68.6 & 337 & 71.5 & 0.32 \\
\hline smoker & 148 & 31.4 & 134 & 28.5 & \\
\hline \multicolumn{6}{|c|}{ Drinking status } \\
\hline No & 374 & 79.4 & 388 & 82.4 & 0.25 \\
\hline Yes & 97 & 20.6 & 83 & 17.6 & \\
\hline
\end{tabular}

${ }^{\mathrm{a}}$ Two-sided $\chi^{2}$ test. 
Table 2 (on next page)

Single nucleotide polymorphism information and Hardy-Weinberg test. 
1 Table 2 Single nucleotide polymorphism information and Hardy-Weinberg test.

\begin{tabular}{llllllll}
\hline Gene & Position & SNP & Region & $\begin{array}{l}\text { Allele } \\
\text { gene }\end{array}$ & MAF & $\begin{array}{l}\text { Functional } \\
\text { change }\end{array}$ & $\begin{array}{l}P \\
\text { value }\end{array}$ \\
\hline C7 & chr5:40908799 & rs1376178 & promoter & C/A & 0.45 & STAT1 $^{\mathrm{a}}$ & 0.393 \\
C7 & chr5:40981587 & rs1061429 & 3'UTR $^{\prime}$ & C/A & 0.39 & has-miR-591 $^{\mathrm{b}}$ & 0.710
\end{tabular}

2 a Typical transcription factor changes of promoter.

3 b Typical miRNA binding changes of 3'UTR. 
Table 3 (on next page)

Genotype frequencies of $C 7$ and their association with gastric carcinoma . 
1 Table 3 Genotype frequencies of $C 7$ and their association with gastric carcinoma.

\begin{tabular}{|c|c|c|c|c|c|c|}
\hline \multirow{2}{*}{ Genotypes } & \multicolumn{2}{|c|}{ Controls $(n=471)$} & \multicolumn{2}{|c|}{$\operatorname{Cases}(n=471)$} & \multirow{2}{*}{$O R(95 \% \mathrm{CI})^{\mathrm{a}}$} & \multirow{2}{*}{$P$ value } \\
\hline & $\mathrm{N}$ & $(\%)$ & $\mathrm{N}$ & $(\%)$ & & \\
\hline \multicolumn{7}{|c|}{ C7 rs 1376178} \\
\hline $\mathrm{CC}$ & 130 & 27.6 & 77 & 16.4 & 1.00ref & \\
\hline $\mathrm{CA}$ & 226 & 48.0 & 252 & 53.5 & $1.88(1.35-2.63)$ & $<0.001$ \\
\hline AA & 115 & 24.4 & 142 & 30.1 & $2.09(1.43-3.03)$ & $<0.001$ \\
\hline \multicolumn{7}{|c|}{ C7 rs 1061429} \\
\hline $\mathrm{CC}$ & 246 & 52.2 & 225 & 47.8 & 1.00ref & \\
\hline $\mathrm{CA}$ & 191 & 40.6 & 179 & 38.0 & $1.03(0.78-1.35)$ & 0.861 \\
\hline $\mathrm{AA}$ & 34 & 7.2 & 67 & 14.2 & $2.16(1.37-3.38)$ & 0.001 \\
\hline
\end{tabular}

2 adjusted for age, gender smoking status and drinking status. 


\section{Table 4 (on next page)}

Association of C7 rs1376178 C > A polymorphism with GC risk stratified by selected variables. 
1 Table 4 Association of $C 7$ rs $1376178 \mathrm{C}>\mathrm{A}$ polymorphism with $\mathrm{GC}$ risk stratified by selected 2 variables.

\begin{tabular}{|c|c|c|c|c|c|}
\hline \multirow[t]{2}{*}{ Variables } & \multicolumn{3}{|c|}{ Genotypes (Controls/Cases) } & \multirow{2}{*}{$\begin{array}{l}\text { AA/CC model } \\
\text { OR }(95 \% \mathrm{CI})^{\mathrm{a}}\end{array}$} & \multirow[t]{2}{*}{$P$ value } \\
\hline & $\mathrm{CC}$ & $\mathrm{CA}$ & $\mathrm{AA}$ & & \\
\hline \multicolumn{6}{|l|}{ Gender } \\
\hline Male & $91 / 48$ & $158 / 176$ & $72 / 108$ & $2.88(1.81-4.58)$ & $<0.001$ \\
\hline Female & $39 / 29$ & $68 / 76$ & $43 / 34$ & $1.06(0.55-2.06)$ & 0.862 \\
\hline \multicolumn{6}{|l|}{ Age } \\
\hline$<60$ & $66 / 40$ & $97 / 108$ & $46 / 61$ & $2.19(1.26-3.78)$ & 0.005 \\
\hline$\geq 60$ & $64 / 37$ & $129 / 144$ & $69 / 81$ & $2.07(1.23-3.48)$ & 0.006 \\
\hline \multicolumn{6}{|c|}{ Smoking status } \\
\hline No & $92 / 51$ & $156 / 172$ & $89 / 100$ & $2.05(1.31-3.21)$ & 0.002 \\
\hline Yes & $38 / 26$ & $70 / 80$ & $26 / 42$ & $2.40(1.19-4.87)$ & 0.015 \\
\hline \multicolumn{6}{|c|}{ Drinking status } \\
\hline No & $105 / 62$ & $181 / 196$ & $102 / 116$ & $1.94(1.28-2.93)$ & 0.002 \\
\hline Yes & $25 / 15$ & $45 / 56$ & $13 / 26$ & $3.55(1.37-9.17)$ & 0.009 \\
\hline
\end{tabular}

3 a Data were calculated by unconditional logistic regression and adjusted for age, gender, smoking 4 and drinking status, where they were appropriate. 


\section{Table 5 (on next page)}

Association of C7 rs1061429C > A polymorphism with GC risk stratified by selected variables. 
1 Table 5 Association of $C 7$ rs $1061429 \mathrm{C}>$ A polymorphism with GC risk stratified by selected 2 variables.

\begin{tabular}{|c|c|c|c|c|c|}
\hline \multirow[t]{2}{*}{ Variables } & \multicolumn{3}{|c|}{ Genotypes (Controls/Cases) } & \multirow{2}{*}{$\begin{array}{l}\text { AA/CC model } \\
\text { OR }(95 \% \mathrm{CI})^{\mathrm{a}}\end{array}$} & \multirow[t]{2}{*}{$P$ value } \\
\hline & $\mathrm{CC}$ & $\mathrm{CA}$ & $\mathrm{AA}$ & & \\
\hline \multicolumn{6}{|l|}{ Gender } \\
\hline Male & $172 / 165$ & $125 / 123$ & $24 / 44$ & $1.94(1.13-3.33)$ & 0.017 \\
\hline Female & $74 / 60$ & $66 / 56$ & $10 / 23$ & $2.87(1.26-6.54)$ & 0.012 \\
\hline \multicolumn{6}{|l|}{ Age } \\
\hline$<60$ & $110 / 106$ & $87 / 70$ & $12 / 33$ & $2.84(1.39-5.80)$ & 0.004 \\
\hline$\geq 60$ & $136 / 119$ & $104 / 109$ & $22 / 34$ & $1.81(1.00-3.28)$ & 0.050 \\
\hline \multicolumn{6}{|c|}{ Smoking status } \\
\hline Non-smoker & $172 / 149$ & $142 / 119$ & $23 / 55$ & $2.79(1.63-4.77)$ & $<0.001$ \\
\hline smoker & $74 / 76$ & $49 / 60$ & $11 / 12$ & $1.03(0.43-2.5)$ & 0.947 \\
\hline \multicolumn{6}{|c|}{ Drinking status } \\
\hline No & $203 / 180$ & $154 / 141$ & $31 / 53$ & $1.92(1.18-3.13)$ & 0.008 \\
\hline Yes & $43 / 45$ & $37 / 38$ & $3 / 14$ & $5.23(1.34-20.40)$ & 0.017 \\
\hline
\end{tabular}

3 a Data were calculated by unconditional logistic regression and adjusted for age, gender, smoking 4 and drinking status, where they were appropriate. 
Table 6(on next page)

Table 6 Correlation between $C 7$ polymorphisms and clinical characteristics 
1 Table 6 Correlation between $C 7$ polymorphisms and clinical characteristics

\begin{tabular}{ccccc}
\multirow{2}{*}{$\begin{array}{c}\text { Clinical pathological } \\
\text { characteristics }\end{array}$} & \multicolumn{2}{c}{ rs1061429 C $>\mathrm{A}$} & \multicolumn{2}{c}{ rs1376178 C $>\mathrm{A}$} \\
\cline { 2 - 5 } & Tau-b & $P$ value & Tau-b & $P$ value \\
\hline TNM stage & 0.074 & 0.089 & 0.021 & 0.631 \\
Tumor size & 0.071 & 0.123 & -0.004 & 0.935 \\
Lymph node metastasis & 0.104 & 0.022 & 0.065 & 0.156 \\
Distant metastasis & 0.086 & 0.075 & 0.027 & 0.569 \\
\hline
\end{tabular}

2 


\section{Figure 1}

Fig. 1 C7 expression in gastric cancer and its effect on the prognosis of gastric cancer patients

(A) C7 expression in gastric cancer and adjacent normal tissues in GEPIA database. $(* P<$ 0.05). (B) C7 expression in serum of normal individuals and gastric cancer patient. (C) Overall survival and (D) post-progression survival analysis of gastric cancer patients based on C7 expression. 

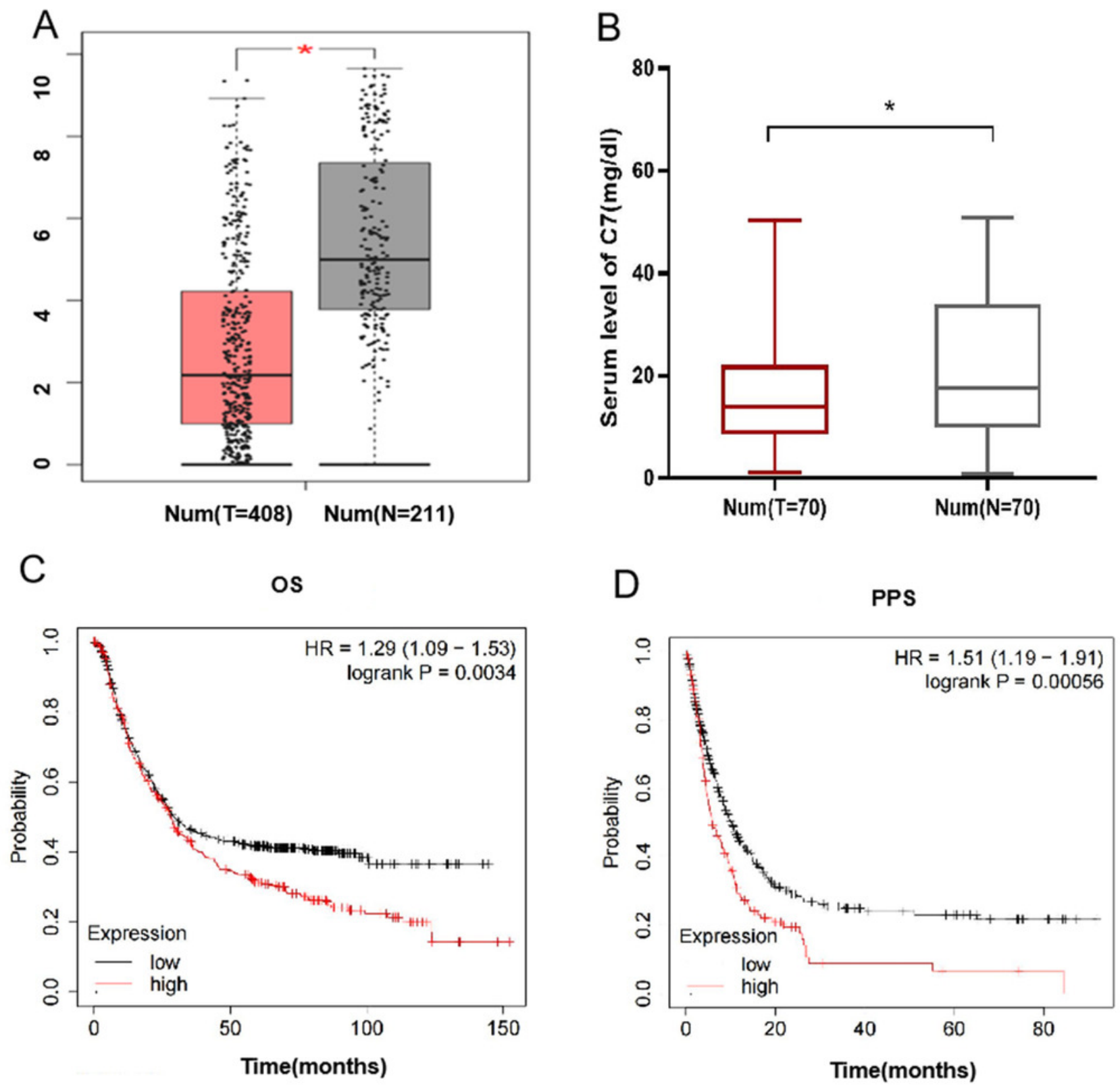
Figure 2

Fig. 2 Association of $\mathrm{C} 7$ expression in gastric cancer patients with $\mathrm{C} 7$ genetic polymorphisms

(A) rs1061429 genotypes from GTEx database. (B) rs1061429 genotypes and (C) rs1376178 genotypes in serum.

A

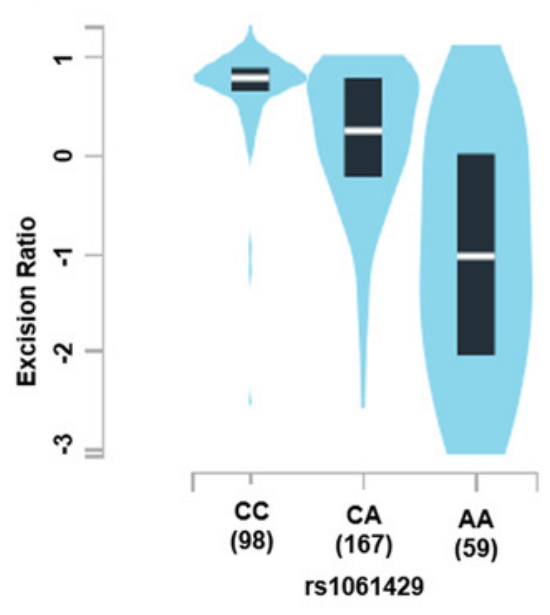

B

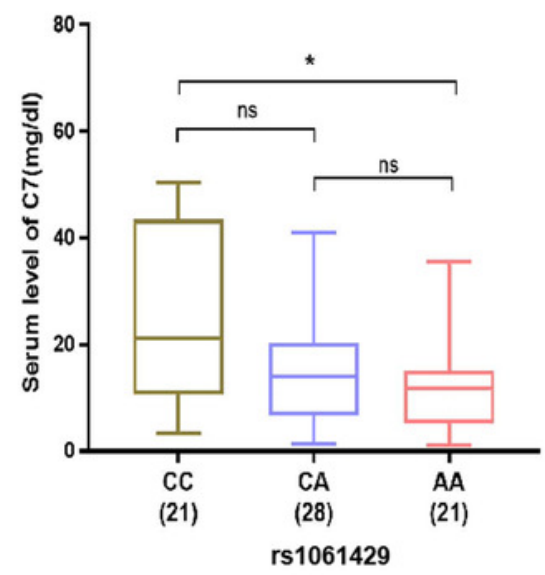

C

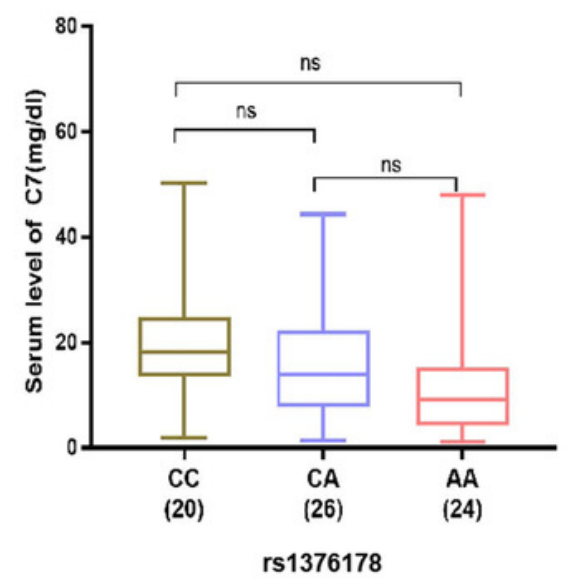


Figure 3

Fig 3. The effect of $\mathrm{C} 7$ polymorphisms on transcription activity

(A) Different allele of rs 1376178 had different regulatory effects in BGC823 cells. (B) Different allele of rs1061429 had different 3'UTR activity in BGC823 cells. ${ }^{* * * P}<0.001 ; * * P<0.01$.
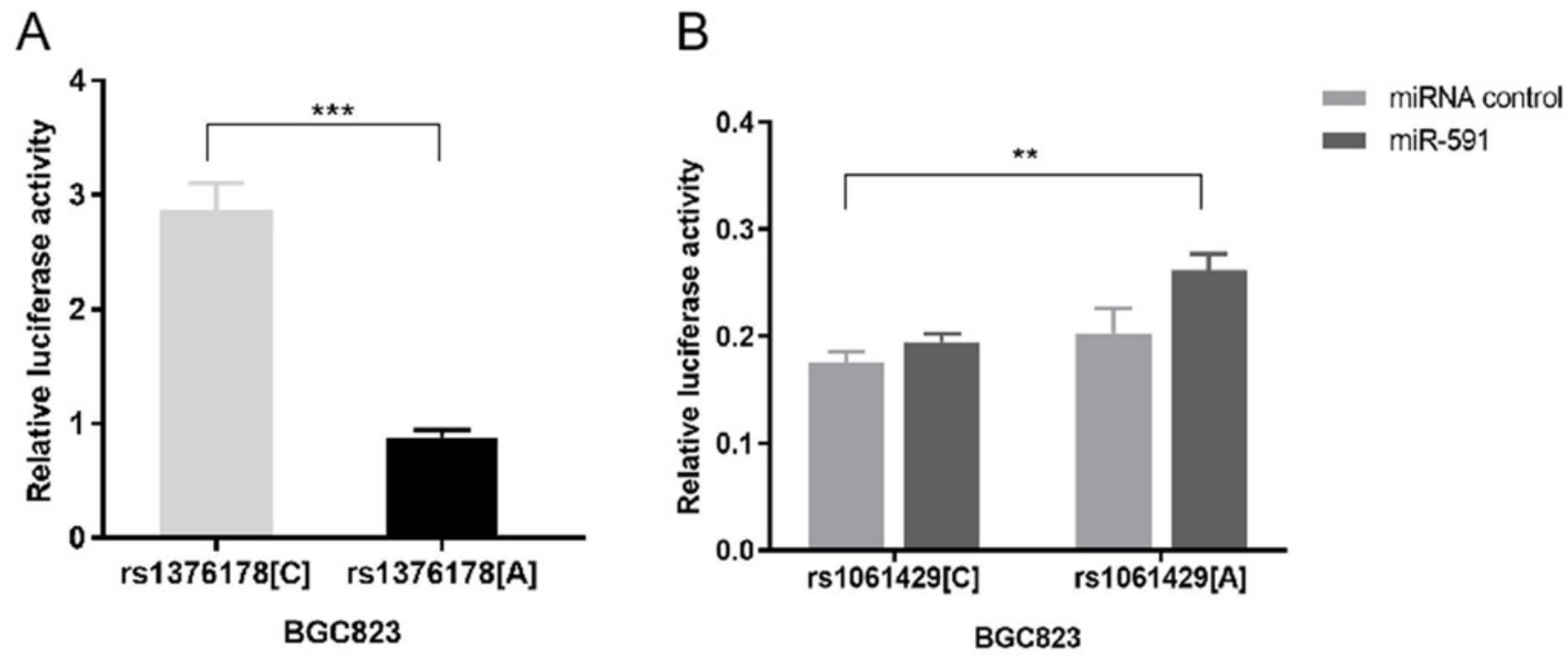